ARTICLE

https://doi.org/10.1038/s41467-018-07851-1

\title{
Lattice engineering enables definition of molecular features allowing for potent small-molecule inhibition of HIV-1 entry
}

Yen-Ting Lai (1) ${ }^{1}$, Tao Wang ${ }^{2}$, Sijy O'Dell ${ }^{1}$, Mark K. Louder (1) ${ }^{1}$, Arne Schön ${ }^{3}$, Crystal S. F. Cheung ${ }^{1}$, Gwo-Yu Chuang ${ }^{1}$, Aliaksandr Druz ${ }^{1}$, Bob Lin ${ }^{1}$, Krisha McKee ${ }^{1}$, Dongjun Peng ${ }^{1}$, Yongping Yang ${ }^{1}$, Baoshan Zhang ${ }^{1}$, Alon Herschhorn ${ }^{4,5}$, Joseph Sodroski ${ }^{4}$, Robert T. Bailer ${ }^{1}$, Nicole A. Doria-Rose ${ }^{1}$, John R. Mascola', David R. Langley ${ }^{6}$ \& Peter D. Kwong ${ }^{1}$

Diverse entry inhibitors targeting the gp120 subunit of the HIV-1 envelope (Env) trimer have been developed including BMS-626529, also called temsavir, a prodrug version of which is currently in phase III clinical trials. Here we report the characterization of a panel of smallmolecule inhibitors including BMS-818251, which we show to be $>10$-fold more potent than temsavir on a cross-clade panel of 208-HIV-1 strains, as well as the engineering of a crystal lattice to enable structure determination of the interaction between these inhibitors and the HIV-1 Env trimer at higher resolution. By altering crystallization lattice chaperones, we identify a lattice with both improved diffraction and robust co-crystallization of HIV-1 Env trimers from different clades complexed to entry inhibitors with a range of binding affinities. The improved diffraction reveals BMS-818251 to utilize functional groups that interact with gp120 residues from the conserved $\beta 20-\beta 21$ hairpin to improve potency.

\footnotetext{
${ }^{1}$ Vaccine Research Center, NIAID, National Institutes of Health, Bethesda, MD 20892, USA. ${ }^{2}$ Departments of Discovery Chemistry and Molecular Technologies, Bristol-Myers Squibb Research and Development, Cambridge, MA 02142, USA. ${ }^{3}$ Department of Biology, Johns Hopkins University, Baltimore, MD 21218, USA. ${ }^{4}$ Department of Cancer Immunology and Virology, Dana-Farber Cancer Institute, Harvard Medical School, Boston, MA 02215, USA. ${ }^{5}$ Division of Infectious Diseases and International Medicine, Department of Medicine, University of Minnesota, Minneapolis, MN 55455, USA. ${ }^{6}$ Computer

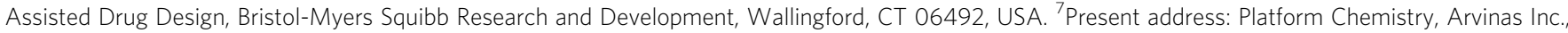
New Haven, CT 06511, USA. Correspondence and requests for materials should be addressed to P.D.K. (email: pdkwong@nih.gov)
} 
T he entry of HIV-1 into target cells is a critical event in the viral life cycle and a target for drug development ${ }^{1}$. Viral entry is mediated by the HIV-1 envelope (Env) glycoprotein trimer, a type 1 fusion machine comprised of three gp120 subunits and three noncovalently linked gp41 subunits, which binds to cell-surface receptors and fuses viral and host cell membranes ${ }^{2}$. Entry inhibitors targeting the gp120 subunit have been developed ${ }^{3}$, with a promising small-molecule lead, fostemsavir, the prodrug version of BMS-626529 (temsavir) currently in phase III clinical trials ${ }^{4}$. Notwithstanding its advanced development and novel mode of action, next-generation inhibitors of fostemsavir have been sought to improve ADME (absorption, metabolism, distribution and elimination) profile ${ }^{5}$, to overcome expected drug resistance ${ }^{6}$, and to increase potency. We note that these qualities may be related; for example, increasing the potency of an inhibitor can be an effective way to counter drug resistance ${ }^{7}$, as resistance mutations generally have only incremental effects on the dose-response of a drug 8 .

$\mathrm{X}$-ray crystallography is often instrumental in determining drug-binding mode and in facilitating structure-based drug design $^{9-11}$. However, structure-based drug design can only be reliably carried out with crystals that diffract with resolution sufficient to provide accurate structural models; unfortunately, this resolution prerequisite has been difficult to achieve for many drug targets, even with extensive screening of crystallization conditions and protein variants ${ }^{12}$. Crystal engineering 13,14 represents an alternative strategy for crystal improvement, whereby inspection of a lattice with poor diffraction identifies weak lattice contacts, which can then be altered through structure-based design. However, both of these strategies can inadvertently introduce modifications that change the properties of protein targets and even their structures ${ }^{15,16}$.

Crystallization chaperones, such as antibody fragments, have also been used to facilitate formation of crystal lattice contacts for difficult protein targets ${ }^{17}$. We recently reported the structure of BMS-626529 (temsavir) in complex with an HIV-1 Env trimer bound by crystallization chaperones comprising the antigenbinding fragments (Fabs) of antibodies 35O22 and PGT122 (ref. ${ }^{18}$ ). We also reported the structure of BMS-378806 (ref. ${ }^{18}$ ), the prototype small molecule for this class of compounds, in the same Env-35O22-PGT122 lattice. In both cases, the resolution was only $3.8 \AA$, and there was uncertainty in the positioning of small-molecule atoms and in the definition of side-chain interactions.

To obtain structural information of improved accuracy, we test a strategy involving the lattice-based engineering of crystallization chaperones. This strategy provides a way to improve a lattice without altering the protein target. We engineer crystallization chaperones to identify a crystal lattice suitable for determining highresolution structures of inhibitors, spanning a range of $>6000$-fold neutralization potency, in complex with envelope trimers of clade A and B HIV-1 strains. We use this lattice to examine small-molecule inhibitors related to BMS-626529 and report structures of multiple small-molecule inhibitors, including that of BMS-818251, an HIV-1 entry inhibitors with $>10$-fold higher potency than BMS-626529, which reveal structural determinants of potent HIV-1 inhibition and provide insights into the design of better entry inhibitors for this class of HIV-1 drugs.

\section{Results}

BMS-818251 shows $>10$-fold increased potency over temsavir. By screening a library of temasvir derivatives, we identified two compounds, BMS-814508 and BMS-818251, which showed improved entry inhibition of the laboratory-adapted HIV-1 strain NL4-3. The $E_{50}$ for BMS-814508 and BMS-818251 was $0.495 \pm$
0.069 and $0.019 \pm 0.003 \mathrm{nM}$, respectively, 4 -fold and $\sim 100$-fold more potent than BMS-626529, which had an $\mathrm{EC}_{50}$ of $2.2 \pm 0.6 \mathrm{nM}$ against the same strain ${ }^{19}$. Both of the improved compounds used a cyano alkene to replace an amide group with different thiazole substituents replacing the triazole on the 6-azaindole core of BMS-626529 (temsavir) (Fig. 1a, Supplementary Fig. 1).

To verify neutralization potency seen in the neutralizationsensitive NL4-3 strain, we further tested the ability of these compounds to inhibit entry on a cross-clade panel of $30 \mathrm{HIV}-1$ strains derived from primary isolates (Fig. 1b). The 30 strains from all major HIV-1 clades were chosen to encompass the complete range of neutralization sensitivity towards BMS-626529 in a 208-strain panel (ref. ${ }^{18}$ ). To provide comparative data, we assessed the well-studied entry inhibitors BMS-378806 and BMS-626529 as controls, and also included BMS-386150, which is very similar to BMS-378806, with a bromine modification in the compound to aid crystal structure determination (Fig. 1a). A recently reported compound $\mathbf{4 8 4}$, which exhibits broad reactivity despite its moderate micro-molar range potency ${ }^{20}$, was also tested. Of note, these six compounds all derive from a piperazine/ piperidine bridge, with various degrees of modification, representing a collection of diverse entry inhibitors targeting the gp120 subunit of the HIV-1 Env trimer (Fig. 1a and Supplementary Fig. 1).

Neutralization of the 30-strain panel generally recapitulated prior observations (Fig. 1b, c). BMS-626529 neutralized this panel of viruses with a geometric mean $\mathrm{IC}_{50}$ of $\sim 0.040 \mu \mathrm{M}$, which was within a factor of three of the geometric mean $\mathrm{IC}_{50}$ of $0.015 \mu \mathrm{M}$ that has been previously reported based on its inhibition of the 208-strain panel ${ }^{18}$. Consistent with the literature, BMS-626529 (temasvir) was substantially more potent than BMS-378806, and compound 484 neutralized 11 of the 30 viruses with micro-molar potency. Despite an overall similarity with BMS-378806, BMS$\mathbf{3 8 6 1 5 0}$ had a substantially weaker ( 7-fold lower) neutralization potency, and BMS-814508 neutralized 28 of the 30 viruses with a mean inhibition similar to that of BMS-626529 (temsavir). Cytotoxicity was observed for BMS-814508 at the highest concentration $(20 \mu \mathrm{M})$ in the neutralization assay (Supplementary Fig. 2). Strikingly, compound BMS-818251 (geometric mean $\mathrm{IC}_{50} \sim 0.002 \mu \mathrm{M}$ ) showed roughly 20 -fold higher neutralization potency than BMS-626529 (geometric mean $\mathrm{IC}_{50} \sim 0.04 \mu \mathrm{M}$ ) on this 30-strain panel (Fig. 1b, c), with more than half of the viruses neutralized in the subnanomolar range.

The superior potency of BMS-818251 observed on the 30 -strain panel prompted us to assess the neutralization potency of this compound on the larger 208-strain panel (Supplementary Table 1). The geometric mean $\mathrm{IC}_{50}$ of BMS-818251 on the 208-strain panel was $0.0015 \mu \mathrm{M}$, in close agreement with the geometric mean $\mathrm{IC}_{50}$ obtained from the 30 -strain panel. When compared to the neutralization potency on the same 208-strain panel, BMS-818251 has a 10.6-fold higher geometric mean $\mathrm{IC}_{50}$ and 18-fold higher median $\mathrm{IC}_{50}$ compared to BMS-626529 (temsavir) (median $\mathrm{IC}_{50} \mathrm{~s}$ were 0.0005 and $0.0090 \mu \mathrm{M}$ for BMS-818251 and BMS-626529, respectively; geometric mean $\mathrm{IC}_{50} \mathrm{~S}$ were 0.0015 and $0.0159 \mu \mathrm{M}$, respectively).

To assess whether the improved potency of BMS-818251 over temsavir was reflected by higher affinity to the HIV-1 Env trimer, we determined the binding affinity of BMS-818251 to HIV-1 Env trimer by isothermal calorimetry (ITC). ITC showed BMS$\mathbf{8 1 8 2 5 1}$ to have an affinity of $0.047 \mu \mathrm{M}$ to BG505 DS-SOSIP.664 Env trimer at $37^{\circ} \mathrm{C}$, which was substantially higher than the $0.402 \mu \mathrm{M}$ affinity of BMS-626529 under the same condition (Supplementary Fig. 3). Thus, the $>10$-fold improved potency of BMS-818251 over temasvir in neutralization assays was associated with an 8.6-fold higher affinity, as determined by the ITC binding assay. 


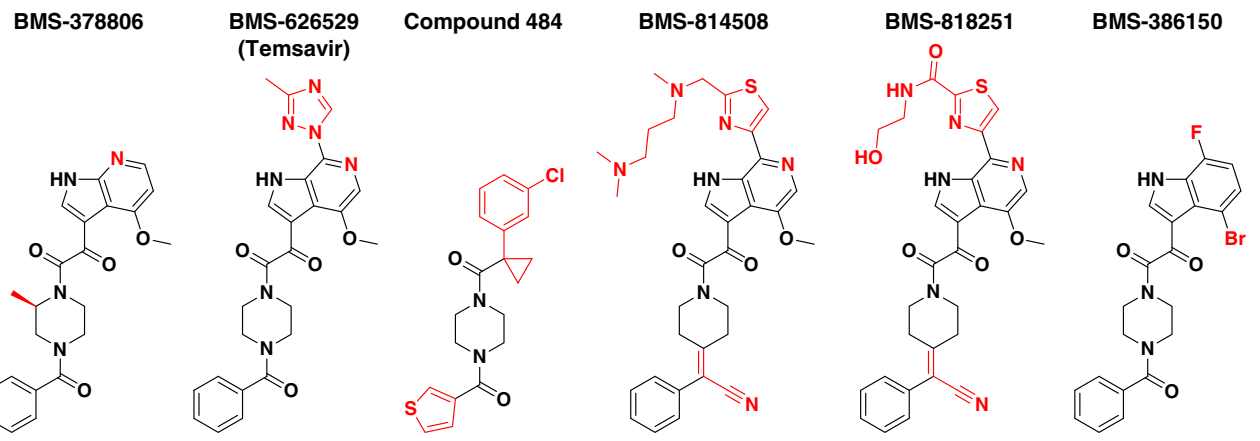

b

\begin{tabular}{|c|c|c|c|c|c|c|c|c|}
\hline Strain & Clade & $\begin{array}{c}\text { BMS- } \\
378806\end{array}$ & $\begin{array}{c}\text { BMS- } \\
626529 \\
\end{array}$ & $\begin{array}{c}\text { Compound } \\
484 \\
\end{array}$ & $\begin{array}{c}\text { BMS- } \\
814508 \\
\end{array}$ & $\begin{array}{c}\text { BMS- } \\
818251\end{array}$ & $\begin{array}{c}\text { BMS- } \\
386150\end{array}$ & \\
\hline $0330 . v 4 . c 3$ & $\mathbf{A}$ & 3.3900 & 0.1850 & $>20$ & 0.0960 & 0.0040 & $>20$ & \\
\hline BG505.W6M.C2 & A & 0.2250 & 0.0050 & $>20$ & 0.0080 & $<0.0003$ & 4.6700 & \\
\hline KER2008.12 & A & $>20$ & 0.7580 & $>\mathbf{2 0}$ & 0.1650 & 0.0110 & $>20$ & \\
\hline MB201.A1 & A & $>20$ & 0.4740 & $>\mathbf{2 0}$ & 0.1920 & 0.0140 & $>20$ & \\
\hline BJOX028000.10.3 & $\mathrm{AE}$ & $>20$ & $>20$ & $>\mathbf{2 0}$ & $>4$ & 0.7330 & $>20$ & \\
\hline C4118.09 & $A E$ & $>20$ & 6.2100 & $>20$ & 2.0000 & 0.0890 & $>20$ & \\
\hline CNE56 & $\mathrm{AE}$ & $>20$ & $>20$ & $>20$ & $>4$ & 0.4710 & $>20$ & \\
\hline R3265.c6 & AE & $>20$ & 5.8400 & $>\mathbf{2 0}$ & 1.0600 & 0.0320 & $>20$ & \\
\hline 242-14 & AG & 0.4270 & 0.0240 & $>\mathbf{2 0}$ & 0.0150 & $<0.0003$ & 7.0200 & \\
\hline DJ263.8 & AG & $>20$ & 0.0120 & 2.7800 & $<0.0003$ & $<0.0003$ & $>20$ & \\
\hline T253-11 & AG & 0.1930 & 0.0005 & 3.3500 & $<0.0003$ & $<0.0003$ & 0.5410 & \\
\hline T280-5 & AG & 0.0310 & 0.0030 & 11.9000 & 0.0008 & $<0.0003$ & 2.0200 & \\
\hline 7165.18 & B & 0.5260 & 0.0200 & $>20$ & 0.4910 & 0.0130 & $>20$ & \\
\hline B41 & B & 0.0010 & 0.0007 & 1.7300 & 0.0010 & $<0.0003$ & 0.1170 & \\
\hline BL01.DG & B & $>20$ & 12.4000 & $>20$ & 2.8100 & 0.5330 & $>20$ & \\
\hline CNE10 & B & 0.0250 & 0.0020 & 1.9400 & 0.0040 & $<0.0003$ & 0.5090 & \\
\hline PVO.04 & B & 0.0080 & 0.0009 & 7.8700 & 0.0020 & $<0.0003$ & 0.6640 & \\
\hline REJO.67 & B & $>20$ & 0.2050 & $>20$ & 2.7300 & 0.4500 & $>\mathbf{2 0}$ & \\
\hline CH119.10 & $\mathrm{BC}$ & 0.1120 & 0.0050 & $>\mathbf{2 0}$ & 0.0040 & $<0.0003$ & 13.7000 & \\
\hline CNE20 & $\mathrm{BC}$ & $>20$ & 0.8780 & $>20$ & 0.1430 & 0.0060 & $>20$ & \\
\hline $426 c$ & C & 0.4450 & 0.0050 & 13.4000 & 0.0360 & 0.0004 & 6.6200 & \\
\hline CAP210.E8 & C & $>20$ & 0.0660 & $>20$ & 0.4000 & 0.0220 & $>20$ & \\
\hline CAP256.206.C9 & C & 0.0210 & 0.0050 & 13.6000 & $<0.0003$ & $<0.0003$ & 1.0400 & \\
\hline DU156.12 & C & 0.0290 & 0.0020 & 7.6900 & 0.0200 & $<0.0003$ & 1.3500 & IC50 uM \\
\hline ZM106.9 & C & 0.2060 & 0.0160 & $>20$ & 0.0310 & 0.0005 & 10.8000 & $<0.001$ \\
\hline $247-23$ & D & $>20$ & 0.0090 & $>20$ & 0.0160 & $<0.0003$ & $>20$ & $0.001-0.01$ \\
\hline A03349M1.vrc4a & D & 0.8150 & 0.0050 & $>20$ & 0.0080 & $<0.0003$ & $>20$ & $0.01-0.100$ \\
\hline NKU3006.ec1 & D & 0.0120 & 0.0020 & 1.6500 & 0.0010 & $<0.0003$ & 0.2560 & $0.100-1.00$ \\
\hline X1193.c1 & $\mathbf{G}$ & 0.3370 & 0.0310 & 13.6000 & 0.0060 & $<0.0003$ & $>20$ & $1.00-10.0$ \\
\hline P0402.c2.11 & G & 3.6000 & 0.0170 & $>\mathbf{2 0}$ & 0.0120 & $<0.0003$ & $>20$ & $>10.0$ \\
\hline
\end{tabular}

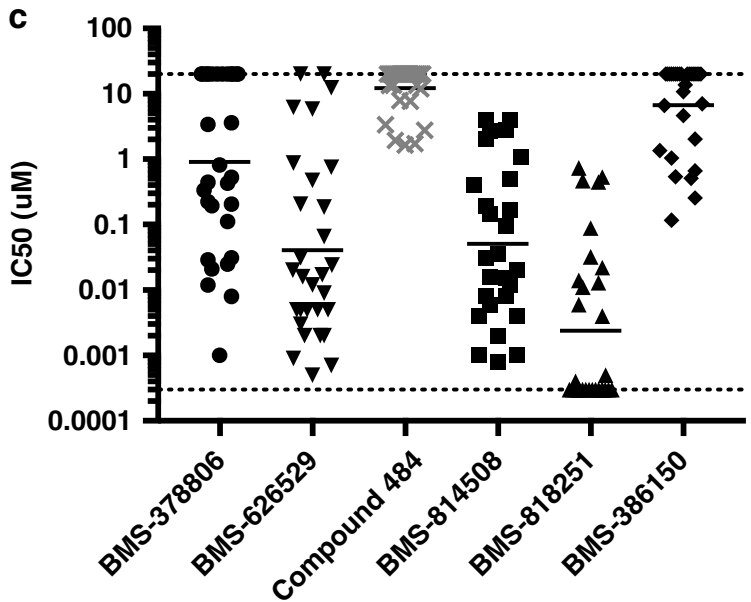

- BMS-378806

$\checkmark$ BMS-626529

$\times$ Compound 484

BMS-814508

A BMS-818251

- BMS-386150

Fig. 1 Diverse HIV-1 entry inhibitors span >6000-fold differences in neutralization potency, with BMS-818251 being >20-fold more potent than BMS-626529 (temasvir). a HIV-1 entry inhibitors with common functional groups shown in black and unique features in red. $\mathbf{b}$ Neutralization assay of entry inhibitors against thirty HIV-1 isolates from all major HIV-1 clades. c Neutralization data shown as a scatter plot, with the geometric mean shown as horizontal bars. Dotted lines show the detection limits of neutralization assay 
Lattice engineering enables higher-resolution structures. To provide a structure-function relationship between these smallmolecule leads and their HIV-1 target, we sought to crystallize these compounds with the HIV-1 Env trimer. Previously we determined cocrystal structures of BMS-626529 and BMS-378806 at a resolution of $3.8 \AA$, bound to the BG505 SOSIP.664 trimer along with two crystallization chaperones, Fab $35 \mathrm{O} 22$ and PGT122 (ref. ${ }^{18}$ ). The BG505 SOSIP.664 Env trimer is a near-native Env trimer comprising fully cleaved gp120 and gp41 subunits, derived from strain BG505, with stabilized disulfide (SOS) and I559P (IP) mutation and truncated at residue 664 (ref. ${ }^{21}$ ). These cocrystal structures defined the general pose of drug compounds in the gp120 binding pocket, but left uncertain the atomic position of chemical substituents. To improve the resolution of the diffraction from this lattice (Fig. 2a, b), which allowed determination of several Env structures in independent studies (Supplementary Table 2), we attempted to reduce lattice flexibility in the crystallization chaperones (Fab 35O22 and Fab PGT122). The constant region of the $35 \mathrm{O} 22 \mathrm{Fab}$ appeared hyperflexible as judged by its high B-factor (Supplementary Fig. 4a). Removal of the constant region by converting the Fab 35O22 to an $\mathrm{scFv}$ led to crystals that retained the original lattice, with almost identical lattice parameters (Supplementary Table 3). In structures of the $35 \mathrm{O} 22 \mathrm{scFv}$, we observed some regions to be more flexible than in the Fab context, mainly due to a lack of structural support of antibody constant regions. To mitigate this flexibility, we tested two replacement variants: (i) replacing residues ${ }^{10} \mathrm{ELK}^{12}$ with $\beta$-strand-preferring residues ${ }^{10} \mathrm{TTT}^{12}$; (ii) altering $\mathrm{A} 16 \mathrm{~S}$ and $\mathrm{F} 84 \mathrm{~S}$ to reduce exposed hydrophobic atoms; named 35O22_3T2S as in Supplementary Fig. 4b. Crystals generated with $35 \mathrm{O} 22$ _3T2S has a substantially lower Wilson B factor of $28 \AA^{2}$ compared to $86 \AA^{2}$ for the original crystal (Fig. 2c). The refined structure of scFv 35O22_3T2S showed an average B-factor of $71 \AA^{2}$ vs. $202 \AA^{2}$ for the $35 \mathrm{O} 22 \mathrm{Fab}$ in the previous BMS-626529 complexed structure. In addition to mutations introduced into $35 \mathrm{O} 22$, two methionine mutations (MM) were introduced onto the Fab of 3H109L, a precursor of PGT122 found to crystallize better ${ }^{22}$. These methionine mutations potentially interact with the base of gp41 molecules, one layer higher in the lattice (Fig. 2b, d). Finally, two potential glycosylation sites were introduced onto the $35 \mathrm{O} 22 \mathrm{scFv}$, which enhanced its production yield by tenfold (Supplementary Fig. 4b).

To assess the impact of our lattice engineering, we determined the structure of BMS-378806 in the improved lattice with the modified antibody fragments (termed 35O22_3T2S and 3H109L_MM). We observed almost identical unit cell dimensions as the original lattice ${ }^{18}$, but with superior diffraction (Fig. $2 \mathrm{c}$ and Supplementary Table 3 ). Along the three principle directions $a^{*}$, $b^{*}$, and $c^{*}$, diffraction improved from $5.1,4.8$, and $3.0 \AA$ to 3.3 , 3.1 , and $2.3 \AA$, respectively, as judged by the UCLA anisotropy server $^{23}$, with the unique reflections increasing from 22,236 to 51,309 (Fig. 2c). Because the space group $\left(\mathrm{P}_{3}\right)$ remained the same and the unit cell dimensions were almost identical, the effective information content and data-to-parameter ratio obtained from the new crystal was more than double that of the parent lattice (PDB code: 4TVP) (Supplementary Table 3). The resulting electron density maps showed superior density for many regions of the Env trimer. For example, residues 65-73 in the constant region 1 (C1) of gp120 could now be accurately modeled with histidine residues at positions 66 and 72 forming an unusual stacking interaction, which was modeled differently in prior structures (Supplementary Fig. 5). Another region of improved structure modeling occurred at the $\alpha 9$ helix of gp41, where weak electron density previously made placement of amino acid side chains ambiguous ${ }^{22,24,25}$; the clear density in the new structure allowed unambiguous placement of amino acid side chains and correct registry of backbone atoms (Supplementary Fig. 6). It is important to note that the $\mathrm{C} 1$ region and the $\alpha 9$ helix did not directly contact the small-molecule inhibitor; thus the improved resolution of these regions was a consequence of the improved lattice, not an effect of small-molecule stabilization. We also noted that for very dynamic regions of the Env (such as the inter-helical region of gp41), lattice engineering was unable to overcome the intrinsic flexibility of these regions, and they remained unresolved in our crystal structures. While the real space correlation for BMS-378806 only improved from 0.91 to 0.95, the improved electron density did allow for greater structural certainty in placement of small-molecule atoms such as the methyl on the piperazine ring or the rotameric state of the oxoacetyl moiety. Overall, the engineered lattice allowed for substantially greater certainty in the positioning of both smallmolecule compound and interacting Env-trimer side chains.

Structural determination of compound 484-Env interactions. As a test of the engineered lattice, we chose to examine compound 484, which previously resisted cocrystallization, presumably due to its micro-molar range activity ${ }^{20}$. A binding pose of compound $\mathbf{4 8 4}$ had previously been proposed based on computational docking ${ }^{20}$. We used the engineered lattice to determine the cocrystal structure of $\mathbf{4 8 4}$ with the BG505 SOSIP.664 Env trimer and compared it to the docking simulation. The cocrystal structure (Fig. 3a) was similar to the overall pose of 484 in the docking study, though with notable differences. The thiophene ring essentially flipped $180^{\circ}$ between cocrystal structure and docking pose, a difference supported both by the clear electron density defining $\mathbf{4 8 4}$ in the new lattice and by the strong scattering from sulfur, which served to identify this atom in the thiophene ring. Additionally, the piperazine ring rotated $\sim 45^{\circ}$ leading the acetyl-cyclopropane and chloro-benzene to shift $1.5 \AA$ toward the thiophene ring (Fig. 3a).

Overall, 484 interacted mainly with three gp120 fragments: the C-terminus of al helix (residues 107-117; colored cyan in Fig. 3b); the C-terminal part of the CD4-binding loop and subsequent strands (residues 369-385; colored magenta in Fig. $3 \mathrm{~b}$ ); and the $\beta 20-\beta 21$ hairpin (residues 423-436; colored green in Fig. 3b). Hydrophobic interactions dominated the interface between $\mathbf{4 8 4}$ and the BG505 SOSIP.664 Env trimer (Fig. 3c), with the piperazine ring stacked between two tryptophan residues, W427 of the $\beta 20-\beta 21$ loop and W112 of the al-helix. Two residues outside of the three major fragments, residues V255 and M475, also provided hydrophobic interaction with 484. The cocrystal structure of compound 484 thus demonstrated the ability of the engineered lattice to provide atomic-level detail on the interaction between a low potency, low affinity compound, and the HIV-1 Env trimer.

Tail region of BMS-818251 is critical for potent inhibition. We next investigated whether crystal structures derived from the engineered lattice could provide a structure-function explanation for the very high potency of BMS-818251 against HIV-1 BG505 compared to the closely related BMS-814508 $\left(\mathrm{IC}_{50}\right.$ values $<$ 0.0003 vs. $0.008 \mu \mathrm{M}$ for BMS-818251 and BMS-814508, respectively, against BG505; Fig. 1b). These analogs differ only in the chemistry of their respective tail functional groups, the moieties extending from the thiazole ring (Fig. 1a). Examination of the cocrystal structures of BMS-818251 and BMS-814508 with the BG505 SOSIP.664 Env trimer revealed the tail of BMS-818251 to make hydrophilic interactions with BG505 trimer (Fig. 4a), while the tail of BMS-814508 did not make defined contacts with BG505 trimer (Fig. 4b). Specifically, the tail of BMS-818251 interacts with the side chains of K117, R429 and Q432 of 
a

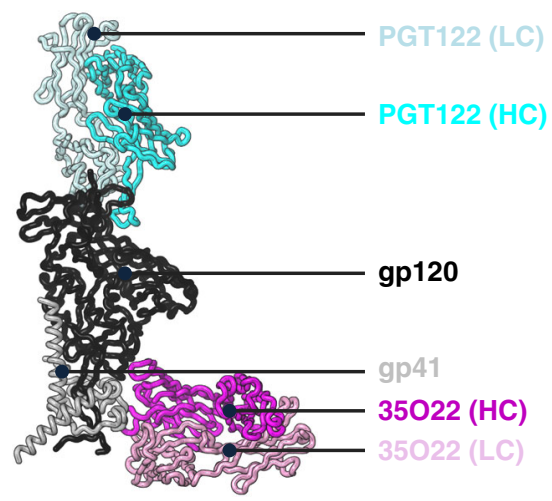

b

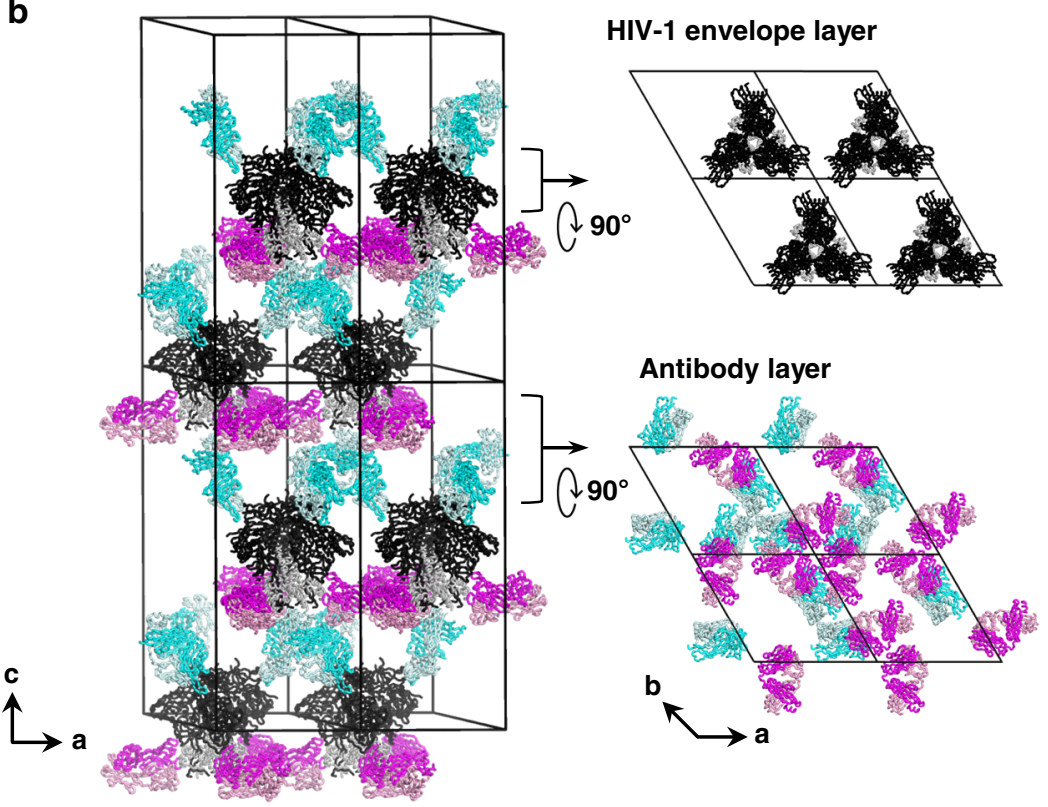

C
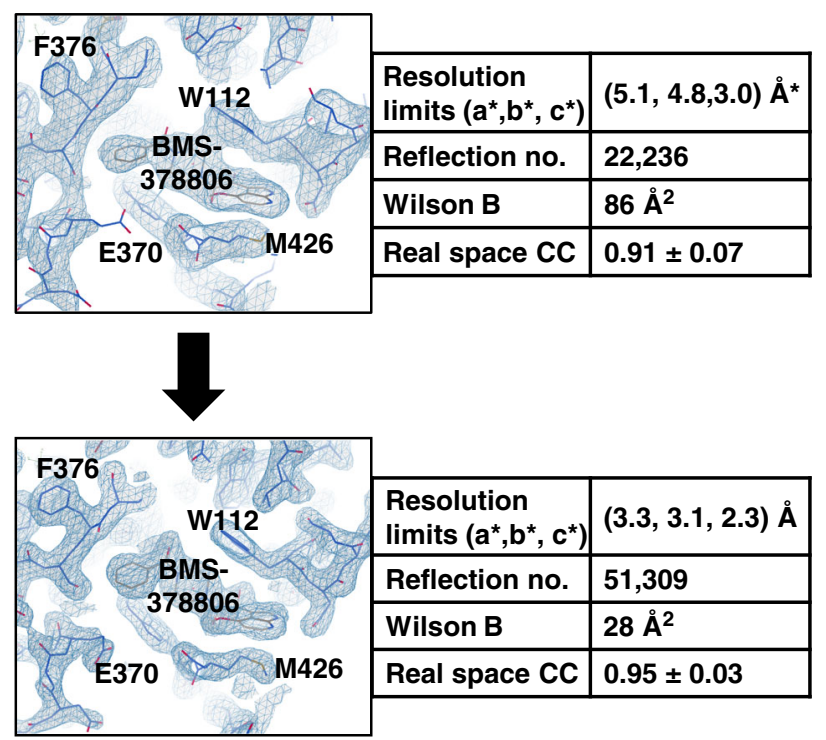

d

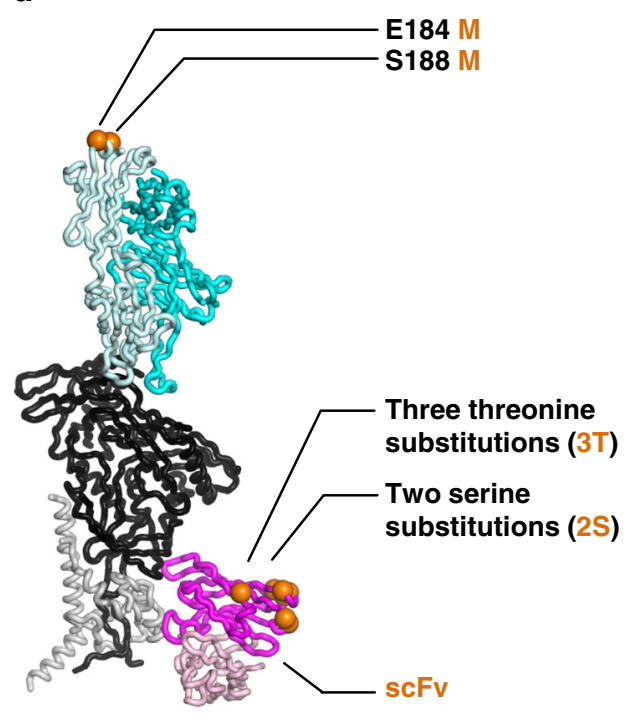

Fig. 2 Molecular assembly of an HIV-1 Env lattice with improved diffraction. a The asymmetric unit of the P6 3 lattice is shown with the PGT122 Fab colored in cyan (HC; heavy chain) and light cyan (LC; light chain); 35022 colored in magenta (HC; heavy chain) and light magenta (LC; light chain). gp120 and gp41 of BG505 SOSIP.664 Env trimer are colored as black and gray respectively. b Crystal packing within the $\mathrm{P}_{3}$ lattice, highlighting the lack of crystal contacts between Env trimers (top right). c Maps and diffraction statistics before and after lattice engineering. 2Fo-Fc electron density maps of the BMS378806 binding pocket, shown as blue mesh, are contoured at $2 \sigma .{ }^{*}$ The original data were truncated with different criteria. For fair comparison, the structure factor was downloaded from the Protein DataBank and the same criteria applied for truncation.) $\mathbf{d}$ Summary of the best changes identified in the antibody fragments to improve the diffraction

BG505 trimer through the terminal hydroxyl group, with clear electron density defining the conformation of the BMS-818251 tail functional group (Fig. 4a; right panel). In addition, residue D113 of Env forms hydrogen bond with the amide nitrogen on the tail of BMS-818251. The lack of electron density surrounding the tail functional group of the highly similar BMS-814508 (Fig. 4b; right panel) suggested that this functional group can adopt multiple conformations and lacks stabilizing interactions with Env.

The difference in the interaction between Env trimer and the tails of BMS-818251 or BMS-814508 likely explains the difference in their neutralization potency. Interestingly, although the chemistry of BMS-818251 and BMS-814508 was identical beyond the tail functional group, we did observe additional interactions between Env residues F382 and E370 with BMS-818251, but not with BMS-814508 (Fig. 4a, b, middle panels). Finally, we observed the piperidine ring of BMS-818251 to adopt a twisted-boat conformation (Fig. 4a, b, left panels), with slightly higher energy than the chair conformation adopted by BMS-814508, suggesting a difference in tail interactions to affect the conformation of the entire compound.

BMS-818251 accommodates residue variations with high potency. BMS-818251 showed superior neutralization potency against the 30-strain panel compared with BMS-814508, ranging 
a
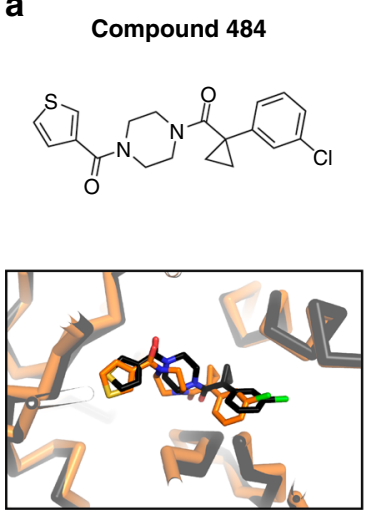
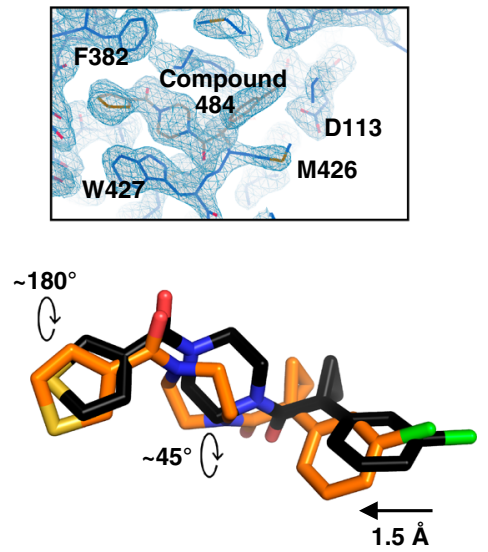

b

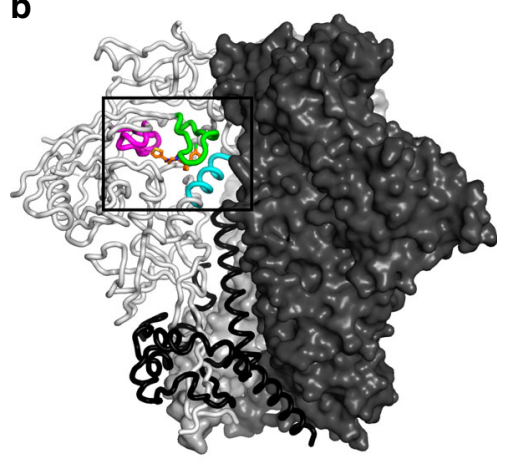

107-117

369-385

423-436

C
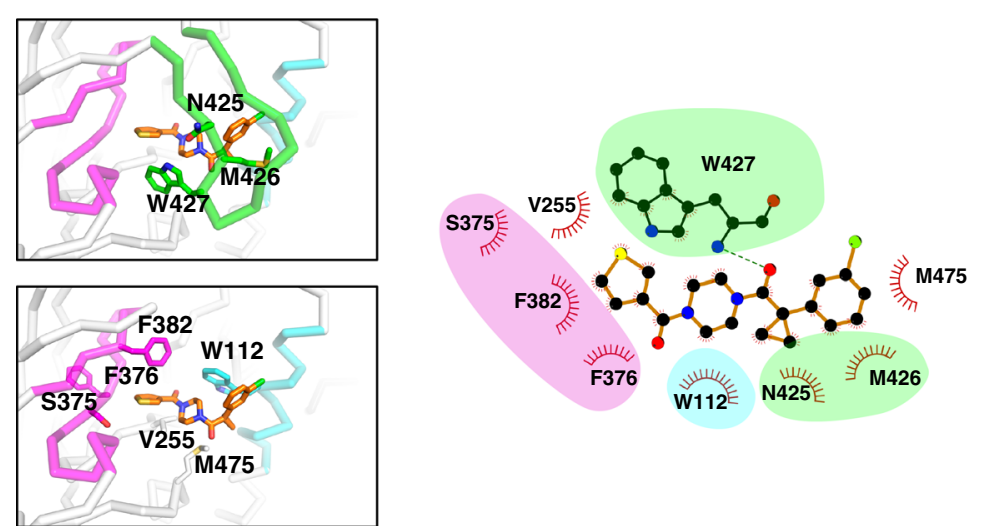

Fig. 3 Chemical definition of compound 484 interaction with HIV-1 Env. a Comparison of cocrystal structure of $\mathbf{4 8 4}$ with docking simulation. The two structures are superimposed by the gp120 (484 not used for superimposition). Simulated $\mathbf{4 8 4}$ complex structure is colored in black and co-crystal structure colored in orange (lower left). 2Fo-Fc electron density showing the $\mathbf{4 8 4}$ binding pocket is contoured at $2 \sigma$ (top right). $\mathbf{b}$ Location of the entry inhibitor binding pocket in the context of HIV-1 Env trimer. The black square indicates the location of the inhibitor binding pocket. Three main protein segments interacting with the inhibitors are highlighted in cyan, magenta and green for residues 107-117, 369-385 and $423-436$ respectively. c Detailed view of the $\mathbf{4 8 4}$ binding site. Upper panel on the left shows the residues of the $\beta 20-\beta 21$ hairpin (green fragment) interacting with $\mathbf{4 8 4}$. Lower panel on the left shows the all other residues interacting with $\mathbf{4 8 4}$ (excluding residues on the $\beta 20-\beta 21$ hairpin, which is removed for clarity). Right, the $\mathbf{4 8 4}$-Env interaction is shown as a 2D plot, with residues belonging to different major segments highlighted in different shades. Hydrophobic interactions are shown as sunray symbols while hydrophilic interactions are shown as dotted lines

from $~ 2.7$ - to 90-fold higher (Supplementary Fig. 7a; excluding three strains, DJ263.8, T253-11, and CAP256.206.C9, that were below the detection limit of assay for both BMS-818251 and BMS-814508). Although the different tails of BMS-818251 and BMS-814508 induced subtle differences throughout their Envbound conformation, we nonetheless focused on Env residues 117,429 , and 432 to provide an explanation for the wide range of improved potency of BMS-818251, because of the direct interaction between these residues and the BMS-818251 tail in the BG505 trimer cocrystal structure (Fig. 4a).

Residue 117 was Lys in all 30 tested HIV-1 strains (except for strain CNE56, where it is a Gln), with multiple residue types at residues 429 and 432; we therefore categorized the fold improvement based on the residue identity in residues 429 and 432 (Supplementary Fig. 7b). With the most prevalent combination of R429 and Q432 (9 out of 30 strains), the improvement in neutralization potency for BMS-818251 ranged from $\sim 2.7$ to $\sim 50$ fold. With G429 and a positively charged residue 432 (K or R; 5 out of 30 strains), the improvement ranged from $\sim 3.3$ to $\sim 53.3$ fold, similar to the R429 and Q432 combination. With negatively charged E429 and a positively charged residue 432 (K or R; 8 out of 30 strains), the fold improvements were similar compared to the combination of R429 and Q432 (ranging from $\sim 3.3$ to 90-fold). Finally, when the residues at 429 and 432 were both positively charged (R429/K432 for REJO.67 and K429/K432 for BL01.DK), the improvement were generally minimal (6.1-fold and 5.3-fold, respectively). Thus a wide range of neutralization sensitivity was observed even with identical or homologous residues at positions 429 and 432 . However, we noted that the neutralization potency of BMS-818251 improved for most of the viruses tested. Overall, the virus neutralization data supported the general benefit of the tail functional group in BMS-818251 compared to its counterpart in BMS-814508, and revealed that improved potency could generally be achieved despite variations at positions 429 and 432, which directly interacted with the BMS-818251 tail functional groups.

Structural basis of clade-dependent neutralization. We sought to examine whether the new lattice could provide experimental feedback on the recognition of Env trimers from different HIV-1 clades. Clade-dependent sensitivity has been observed for the entry inhibitors in the BMS-626529 family ${ }^{18}$. Among the three 
a
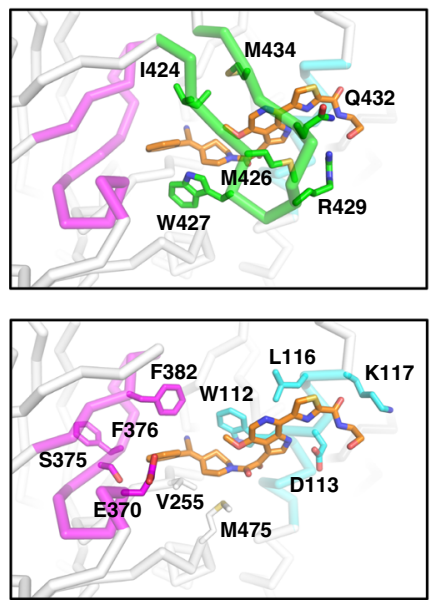

b
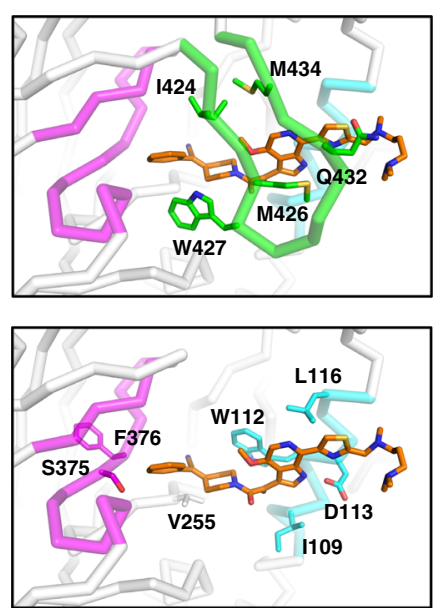

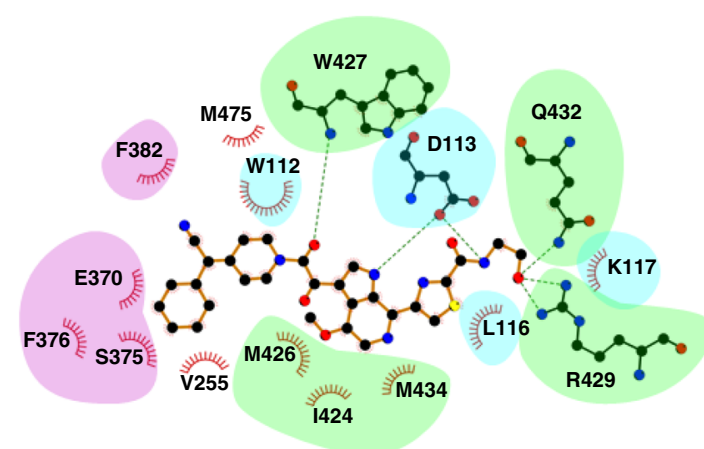

BMS-818251
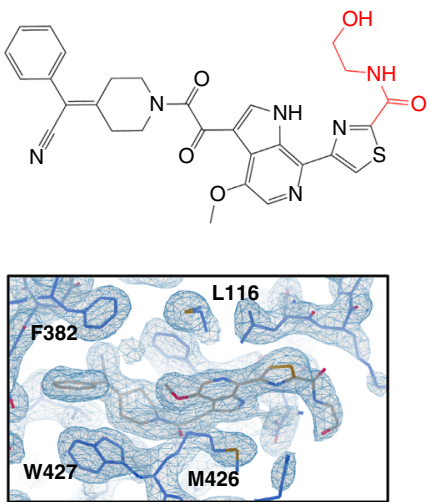

BMS-814508
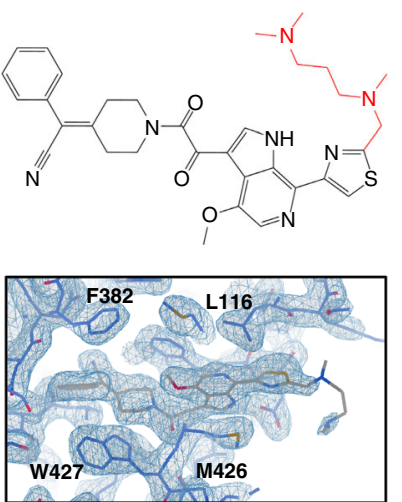

Fig. 4 Crystal structures derived from improved lattice reveal molecular features associated with enhanced neutralization potency. a The complex structure of BMS-818251 with BG505 SOSIP.664 Env trimer is shown in the top panel on the left with interacting residues on the $\beta 20-\beta 21$ hairpin (green segment) shown as sticks and labeled; interacting residues other than those on the $\beta 20-\beta 21$ hairpin are shown as sticks and labeled in the lower left panel. BMS$\mathbf{8 1 8 2 5 1}$ is shown as orange sticks. Middle, BMS-818251 interaction with BG505 SOSIP.664 Env trimer is shown as a 2D plot, with residues belonging to the three major fragments colored accordingly. Top right, the chemical formula of BMS-818251; bottom right, 2Fo-Fc electron density map surrounding the inhibitor binding pocket is shown as blue mesh, contoured at $2 \sigma$. b Crystal structure (left), 2D plot (middle) and electron density map (bottom right) of BMS-814508 (top right) shows the interaction between the inhibitor and BG505 SOSIP.664 Env trimers

major clades that had been well studied with this class of inhibitors, clade $\mathrm{B}$ strains were the most sensitive, followed by clade $\mathrm{C}$ strains with moderate sensitivity and clade A strains with least sensitivity. To investigate the structural attributes responsible for this clade dependency, we first determined the compound-free Env-trimer structure of the B41 strain, a clade B virus with only $78 \%$ sequence identity to the clade A BG505. Residues that differed in sequence between B41 and BG505 were observed to account for $28 \%$ of the Env-trimer surface (Fig. 5a). Surface differences were also present in the epitopes of the crystallization chaperones 35O22_3T2S and 3H109L_MM, but these modified antibodies were able to accommodate these changes, and crystals of the B41 SOSIP.664 Env trimer had unit cell dimensions similar to those of the BG505 SOSIP.664 Env trimer crystals (Fig. 5b).

We next sought to determine cocrystal structures of Envs from HIV-1 strains B41 and BG505 with BMS-386150, a compound similar to the prototypic BMS-378806, though with a bromine modification to aid structure determination (Fig. 1a). Although there were differences in the three major binding fragments (Fig. 5c, colored in cyan, magenta and green respectively, as defined in Fig. 3 b) interacting with BMS-386150, the interacting residues were mostly conserved between B41 and BG505 Envs.
Furthermore, the BMS-386150-interacting residues specific for B41 or BG505 (highlighted in black rectangles in Fig. 5c) were conserved between these two strains (Supplementary Fig. 8). This observation suggested that the clade dependency of entry inhibitors in the temsavir family was not directly caused by sequence variation in the binding pocket, but might rather be a consequence of various degrees of conformational pliability at the inhibitor binding site among the different clades. Indeed, a modest but significant shift of the $\beta 20-\beta 21$ hairpin loop was observed in the BMS-386150-complexed structures of B41 and BG505 Envs (Fig. 5d, upper panel), supporting the idea that conformational changes around the binding pocket, instead of the sequence identity of residues of the binding pocket, dictate the clade-dependent neutralization potency of this class of entry inhibitors. This conclusion was further strengthened by the fact that B41 and BG505 showed conformational differences around the drug-binding pocket in their compound-free structures (Fig. 5d, lower panel).

\section{Discussion}

Crystal engineering has been extensively explored for small molecules crystals ${ }^{26,27}$, such as metal-organic frameworks ${ }^{28,29}$, 
a

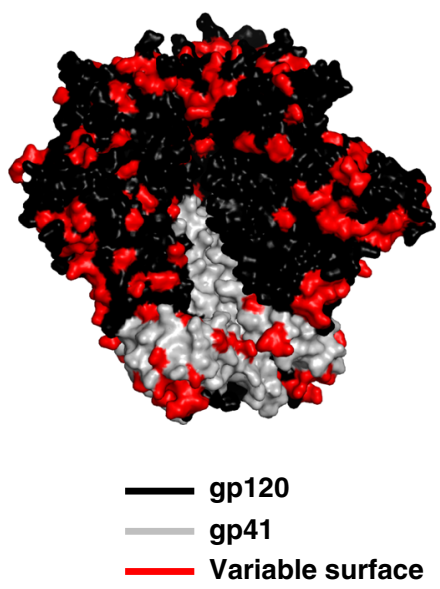

C

BG505/BMS-386150

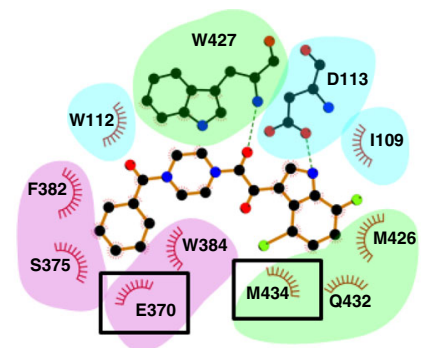

B41/BMS-386150

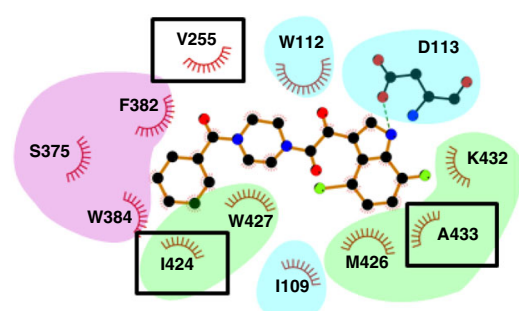

b

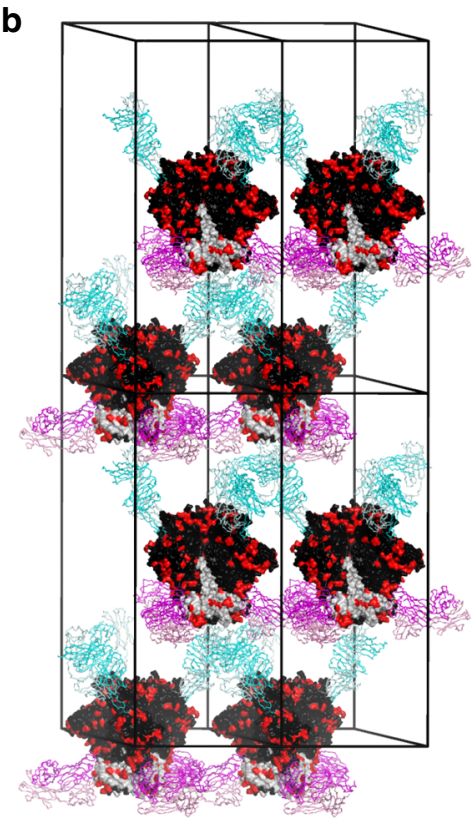

d

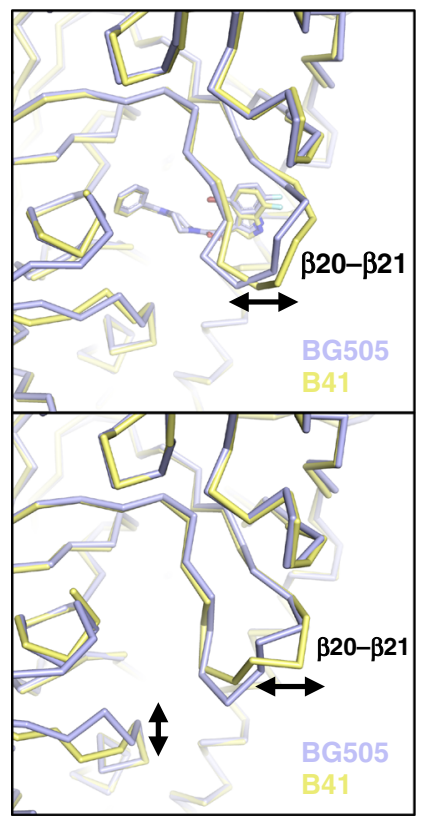

Fig. 5 Generalizable crystallization platform for diverse HIV-1 envelope and drug complexes. a B41 SOSIP.664 Env trimeric structure in surface representation shows gp120 in black and gp41 in gray; the residues that differ between the B41 SOSIP.664 and BG505 SOSIP.664 Env trimers are colored as red. b B41 SOSIP.664 Env trimeric structure is shown in the context of P6 3 lattice. c Structures of BMS-386150 in complex with the BG505 (top) or B41 (bottom) SOSIP.664 Env trimers. Residues interacting with BMS-386150 that are different between B41 and BG505 are highlighted in black box. d The backbone conformational differences in the structures of BMS-386150 complexed with BG505 (purple) or B41 (yellow) are shown as ribbon diagram (top), the same region in the apo-form BG505 and B41 are also shown (bottom)

which has led to crystals with extraordinary properties, including those with lattice cages, capable of holding diverse small molecules ${ }^{30,31}$. Crystals of biological macromolecules may be more difficult to manipulate due to their greater complexity; however, recent advances have allowed crystal engineering of biological crystals of DNA, protein and peptides ${ }^{32-34}$. We report here the engineering of a protein crystal composed of a highly glycosylated trimer and two crystallization chaperones, which were adapted antibody fragments. We focused on modifying the crystallization chaperones, to remove chaperone flexibility, while avoiding alterations to the target protein. Of note, the antibody fragments were resilient to alteration, and for one of the crystallization chaperones (35O22 Fab), we removed half of its weight, while increasing its capacity to facilitate crystallization. The resulting engineered lattice led to higher resolution and yielded twice as many unique measured reflections as the original crystal, while maintaining similar unit cell parameters; this allowed for improved electron density and more accurate 
modeling of small-molecule inhibitors and Env trimers. Overall, the lattice engineering we report is conceptually similar to a retroengineering strategy reported recently for an organic molecular crystal $^{35}$, but has not to our knowledge been used for macromolecular lattice improvement.

Although antibody fragments and other crystallization chaperones are popular tools for crystallizing difficult protein $\operatorname{targets}^{36,37}$, the retrospective engineering of crystallization chaperones has not been extensively explored. More and more protein targets are being crystallized in the presence of crystallization chaperones $^{38,39}$, as many of these crystals diffract poorly due to suboptimal chaperone properties ${ }^{40,41}$, the engineering strategy reported here may represent a straightforward option for improving their diffraction.

The antiviral potency of the fostemsavir inhibitor is known to display HIV-1 clade dependence on neutralization ${ }^{18}$, but the molecular basis for this dependence has not been identified. Interestingly, we observed conformational differences in the interaction of BMS-386150 with Env trimers from clade A BG505 and clade B B41 HIV-1 strains, despite conservation of all Env residues that contacted BMS-386150. Differences between these interactions related to the positioning and orientation of contact residues, which differed despite being identical in sequence. Specifically, we observed the BG505 and B41 Env trimers to adopt slightly different conformations in their respective binding pockets around the $\beta 20-\beta 21$ hairpin loop region. This difference in binding pocket conformation led to different conserved residues interacting with the inhibitor, manifested as clade dependency in neutralization assays. Our results thus provide insight into the complexity of clade dependence and reveal the importance of obtaining experimental information on small molecules in complex with target Env trimers from diverse clades. The crystal structures of BMS-386150 also provided a basis for its reduced potency, as the bromine substitution, despite being beneficial for structure determination, abrogated the hydrophobic interactions between I424, M426 and M434 and the methoxy functional group that was replaced.

Despite the general importance of clade dependency, we note that the tail functional group that endowed high potency to BMS818251 could accommodate variations on interacting residues at positions 429 and 432 of the HIV-1 Env. The effects of variation in these residues on the enhanced potency of BMS-818251 did not show clade dependency (Supplementary Fig. 7b). The flexibility of the tail functional group of BMS-818251, which can adopt different conformations through torsional angle freedom, may allow it to accommodate the different conformations of the $\beta 20-\beta 21$ structural element in different clades. Furthermore, we note that the BMS-818251 tail contains two functional groups that can be either hydrogen-bond donors or acceptors, potentially increasing its versatility to interact with HIV-1 Env, while the BMS-814508 tail contains two nitrogens that can only be hydrogen-bond acceptors. Thus, multiple ways may exist for small molecules to succumb to or to overcome clade dependency.

ITC binding data showed that BMS-818251 has a $\sim 10$-fold higher affinity to BG505 DS-SOSIP.664 trimer compared to BMS-626529 binding, which is in good agreement with the overall $>10$-fold improved neutralization potency of BMS-818251. However, we note that the neutralization potency is higher than the binding affinity measured by ITC (for example, the BG505 virus was neutralized at $0.0003 \mu \mathrm{M}$ by BMS-818251, while the ITC-determined binding affinity between BMS-818251 and BG505 DS-SOSIP.664 Env trimer was $0.047 \mu \mathrm{M}$ ). This discrepancy is likely because BG505 DS-SOSIP.664 Env trimer is not a perfect mimic of the native BG505 Env trimer on the virion surface. Nonetheless, the fold improvement in affinity correlated well with the fold improvement in neutralization. Thus, BG505
DS-SOSIP.664 Env trimer, despite being an imperfect mimic of native Env, can be a valuable surrogate for the evaluation of improved next-generation entry inhibitors.

It is noteworthy that BMS-818251 exhibited higher antiviral potency than BMS-814508, a related compound that differs only in the tail functional group, for all 30 viruses (excluding 3 viruses that were below the detection limit of assay) tested in this study (Supplementary Fig. 7). AE strains generally are less sensitive to temsavir but were sensitive to BMS-818251, suggesting that BMS-818251 could potentially be pan-reactive to HIV-1 Env. Indeed, we observed a general improvement for BMS-818251 across all major HIV-1 clades, likely enabled by its novel tail functional group. Further optimization of this tail functional group may lead to inhibitors with greater resistance to viral escape. We note that the prodrug version of temsavir is one of the few gp120-directed therapeutics being assessed clinically, with gp120-reactive antibodies like antibody VRC01 in clinical assessment showing lower breadth and potency than temsavir (Supplementary Fig. 9). Overall, the structure of the BMS-818251-Env complex enabled by chaperone-based crystal engineering reveals molecular features explaining the superior neutralization potency of this compound and assisting in the rational design of next-generation inhibitors targeting the gp 120 component of the HIV-1 Env.

\section{Methods}

Synthesis of small-molecule entry inhibitors. The BMS entry inhibitors were synthesized based on Supplementary Figure 10. The oxalamides were prepared via acylation of indole with oxalyl chloride followed by coupling of the acid chloride with 1-benzoylpiperazine (ref. ${ }^{42}$ ) or azaindole with methyl chlorooxoacetate in the presence of $\mathrm{AlCl}_{3}$, followed by hydrolysis of the methyl ester and coupling with 1 benzoylpiperazine (ref. ${ }^{43}$ ) or 2-phenyl-2-(piperidin-4-ylidene)acetonitrile. The substitutions on C-7 of 6-azaindole were installed initially via either copper(0)mediated radical reaction or palladium $(0)$-mediated Stille coupling, followed by structural modifications. Inhibitors were characterized by $1 \mathrm{H}-\mathrm{NMR}, 13 \mathrm{C}-\mathrm{NMR}$ and high-resolution mass spectrometry after synthesis; the representative spectra of BMS-818251, the most potent compound reported in this manuscript are shown in Supplementary Fig. 11. Compound 484 were prepared following published protocol $^{20}$ and described here. $N, N$-diisopropyl ethylamine $(0.6 \mathrm{mmol}), 4$ dimethylaminopyridine $(0.03 \mathrm{mmol})$ and $N$-(3-dimethylaminopropyl)- $N^{\prime}$-ethylcarbodiimide hydrochloride $(0.45 \mathrm{mmol})$ were added to a solution of carboxylic acid $(0.3 \mathrm{mmol})$ and amine $(0.3 \mathrm{mmol})$ in anhydrous dichloromethane $(2 \mathrm{ml})$. The reaction was stirred at room temperature for $5 \mathrm{~h}$ and subsequently concentrated in vacuo. The resulting residue was purified by flash chromatography and the purity of Compound $\mathbf{4 8 4}$ was greater than $95 \%$ according to NMR analysis.

HIV-1 neutralization assays. Neutralization was measured using single-round-ofinfection HIV-1 Env-pseudoviruses and TZM-bl target cells (ARP Cat No. \#8129) 44,45 Compounds were prepared at $400 \mu \mathrm{M}$ with $4 \%$ DMSO (dimethyl sulfoxide) in PBS (phosphate-buffered saline) and diluted 20 times as the starting concentration for serial dilution in the neutralization assays. Pseudoviruses of ten strains from HIV-1 clades $\mathrm{A}, \mathrm{B}$, and $\mathrm{C}$ were incubated with serial dilutions of drug compounds, then added to TZM-bl target cells which have a luciferase reporter gene. After incubation at $37^{\circ} \mathrm{C}$ for 2 days, infection of target cells was measured by luciferase activity. Neutralization curves were fit by nonlinear regression using a five-parameter hill slope equation ${ }^{45}$.

Proteins expression and purification. BG505 SOSIP.664 is a construct with modifications that enabled the production of fully cleaved, prefusion-closed, soluble HIV-1 Env trimer ${ }^{21}$. Proteins were purified following published protocols ${ }^{24}$ with modifications described below. For $1 \mathrm{~L}$ of $\mathrm{Gnti}^{-1-}$ cell culture (ATCC Cat No. CRL-3022), 600ug of BG505 SOSIP.664 (with N332 glycan) expression plasmid and $150 \mu \mathrm{g}$ expression plasmid for Furin were diluted in $50 \mathrm{ml}$ Opti-MEM medium (Life Technology). Three milliliters of Turbo293 transfection reagent (Speed BioSystems) was diluted into $50 \mathrm{ml}$ Opti-MEM medium and incubated for $5 \mathrm{~min}$. Diluted transfection reagent was then added into diluted DNA and incubated for $15 \mathrm{~min}$. The transfection reagent and DNA complex were added into $800 \mathrm{ml}$ of $\mathrm{Gnti}^{-1}$ cells at 2 million $/ \mathrm{ml}$. On the second day of transfection, added $80 \mathrm{ml}$ HyClone SFM4HEK293 medium to each flask of 11 cell cultures. Return flasks to shaker incubator at $120 \mathrm{rpm}, 37^{\circ} \mathrm{C}, 9 \% \mathrm{CO}_{2}$ for additional 6 days. Supernatant was affinity-purified over $2 \mathrm{G} 12$ column, eluted by $3 \mathrm{M} \mathrm{MgCl}_{2}$, followed by size exclusion chromatography in $5 \mathrm{mM}$ Hepes and $150 \mathrm{mM} \mathrm{NaCl}$. Variants of $35 \mathrm{O} 22$ and $3 \mathrm{H} 109 \mathrm{~L}$ antibody fragments were produced in $\mathrm{Gnti}^{-/-}$and Expi cells respectively following published protocol ${ }^{24}$. 3H109L variants were produced as Fab, with heavy chain truncated at the end of constant domain 1 and a purification tag containing 
thrombin site, Strep-tag and 6xHis-tag was appended. After tandem purification by Strep column and Ni-NTA column, the tag was removed by thrombin digestion at $4{ }^{\circ} \mathrm{C}$ overnight, followed by size exclusion chromatography. $35 \mathrm{O} 22 \mathrm{scFv}$ has a single $6 \mathrm{xHis}$-tag for purification and the tag is retained after purification. Two artificial glycans introduced to $35 \mathrm{O} 22$ were preserved by not treating with endoglycosidase.

Crystallization and data collection. Purified trimers were mixed with 3H109L Fab variant (with two methionine modifications) and 35O22_scFv variant (with $3 \mathrm{~T}, 2 \mathrm{~S}$ and 2 glycan modifications) in a 1:3.2:3.2 molar ratio (gp140 protomer: Fab:scFv) and incubated overnight at room temperature before being purified by SEC and concentrated to $10-15 \mathrm{mg} / \mathrm{ml}$ for crystallization. Glycans on the Env and antibody fragments were preserved without the treatment of glycosidase. Crystallization conditions were obtained by sparse chemical screening and optimized manually after initial hits were identified. Diffraction data were collected at APS ID22 and processed using HKL200046. The overall resolution was determined as the highest resolution for which the completeness was greater than $50 \%$ and the $I / \sigma I$ was $>2.0$. Data were then truncated by UCLA anisotropy server (https://services.mbi.ucla.edu/anisoscale/).

Structure solution and refinement. The structures of entry inhibitors in complex with BG505 or B41 SOSIP.664 in the presence of crystallization chaperone variants were solved by molecular replacement using Phaser in $\mathrm{CCP} 4{ }^{47}$ using the nondrugcomplex structure as search model, PDB ID $4 \mathrm{TVP}^{24}$. Small molecules were manually fitted using $\mathrm{COOT}^{48}$ and refinement of the structures was done in Phenix ${ }^{49}$ using optimization of X-ray/stereochemistry and X-ray/ADP weight. CIF files for the small molecules were obtained using ELBOW in Phenix. The asymmetric unit of each crystal structures contains one copy of gp140 protomer, one copy of $35 \mathrm{O} 22 \mathrm{scFv}$ and one copy of 3H209L Fab, with one small-molecule inhibitor occupying the gp120 binding pocket (except the B41 apo structure). Simulated annealing Fo-Fc difference omit map (with small-molecule inhibitors omitted during map calculation) confirmed the modeling of small-molecule inhibitors in the binding pocket (Supplementary Fig. 12). Stereo images of the final 2Fo-Fc map, overlaid on the final model, were shown in Supplementary Fig. 13. The refinement statistics are summarized in Supplementary Table 4. Structural figures were made with Pymol$^{50}$.

Binding affinity measurement. The binding of BMS-626529 and BMS-818251 to BG505 DS-SOSIP was studied by ITC using a VP-ITC from MicroCal/Malvern Instruments Ltd. (Northampton, MA). Prior to the experiment, BG505 DS-SOSIP was dialyzed against PBS, pH 7.5. The inhibitors were first dissolved in $100 \%$ DMSO at concentrations of $10 \mathrm{mM}$, which were then diluted into PBS with additional DMSO to their experimental concentrations of $40 \mu \mathrm{M}$ in the presence of $4 \%$ DMSO. Titrations were performed at $37^{\circ} \mathrm{C}$ by injecting $10 \mu \mathrm{l}$ aliquots of the inhibitor solution into the calorimetric cell (volume $\sim 1.4 \mathrm{ml}$ ) containing BG505 DS-SOSIP prepared at $\sim 2.2 \mu \mathrm{M}$ (expressed per protomer) in buffer with $4 \%$ DMSO.

\section{Data availability}

Crystal structure data that support the findings of this study have been deposited in the Protein Data Bank under the accession code 6MTJ, 6MTN, 6MU6, 6MU7, 6MU8, 6MUF and 6MUG. All other data generated or analyzed during this study are included in this published article (and the supplementary files) or are available from the corresponding author on reasonable request.

Received: 10 April 2018 Accepted: 20 November 2018 Published online: 03 January 2019

\section{References}

1. Engelman, A. \& Cherepanov, P. The structural biology of HIV-1: mechanistic and therapeutic insights. Nat. Rev. Microbiol. 10, 279-290 (2012).

2. Brandenberg, O. F., Magnus, C., Regoes, R. R. \& Trkola, A. The HIV-1 entry process: a stoichiometric view. Trends Microbiol. 23, 763-774 (2015).

3. Lu, L., Yu, F., Cai, L., Debnath, A. K. \& Jiang, S. Development of smallmolecule HIV entry inhibitors specifically targeting gp120 or gp41. Curr. Top. Med. Chem. 16, 1074-1090 (2016).

4. Meanwell, N. A. et al. Inhibitors of HIV-1 attachment: the discovery and development of Temsavir and its Prodrug Fostemsavir. J. Med. Chem. 61 62-80 (2018).

5. Kirchmair, J. et al. Predicting drug metabolism: experiment and/or computation? Nat. Rev. Drug Discov. 14, 387-404 (2015).

6. Allen, R. C., Popat, R., Diggle, S. P. \& Brown, S. P. Targeting virulence: can we make evolution-proof drugs? Nat. Rev. Microbiol. 12, 300-308 (2014).
7. Kurt Yilmaz, N., Swanstrom, R. \& Schiffer, C. A. Improving viral protease inhibitors to counter drug resistance. Trends Microbiol. 24, 547-557 (2016).

8. Hughes, D. \& Andersson, D. I. Evolutionary consequences of drug resistance shared principles across diverse targets and organisms. Nat. Rev. Genet. 16, 459-471 (2015).

9. Murray, C. W. \& Rees, D. C. The rise of fragment-based drug discovery. Nat. Chem. 1, 187-192 (2009).

10. Thomas, S. E. et al. Structural biology and the design of new therapeutics: from HIV and cancer to mycobacterial infections: a paper dedicated to John Kendrew. J. Mol. Biol. 429, 2677-2693 (2017).

11. Blundell, T. L. Protein crystallography and drug discovery: recollections of knowledge exchange between academia and industry. IUCrJ 4, 308-321 (2017).

12. Zheng, H., Hou, J., Zimmerman, M. D., Wlodawer, A. \& Minor, W. The future of crystallography in drug discovery. Expert Opin. Drug Discov. 9, 125-137 (2014).

13. Bauman, J. D. et al. Crystal engineering of HIV-1 reverse transcriptase for structure-based drug design. Nucleic Acids Res. 36, 5083-5092 (2008).

14. Yamada, H. et al. 'Crystal lattice engineering,' an approach to engineer protein crystal contacts by creating intermolecular symmetry: crystallization and structure determination of a mutant human RNase 1 with a hydrophobic interface of leucines. Protein Sci. 16, 1389-1397 (2007).

15. Patel, S. B. et al. Lattice stabilization and enhanced diffraction in human $\mathrm{p} 38$ alpha crystals by protein engineering. Biochim. Biophys. Acta 1696, 67-73 (2004).

16. Kalyoncu, S. et al. Effects of protein engineering and rational mutagenesis on crystal lattice of single chain antibody fragments. Proteins 82, 1884-1895 (2014).

17. Koide, S. Engineering of recombinant crystallization chaperones. Curr. Opin Struct. Biol. 19, 449-457 (2009).

18. Pancera, M. et al. Crystal structures of trimeric HIV envelope with entry inhibitors BMS-378806 and BMS-626529. Nat. Chem. Biol. 13, 1115-1122 (2017).

19. Nowicka-Sans, B. et al. In vitro antiviral characteristics of HIV-1 attachment inhibitor BMS-626529, the active component of the prodrug BMS-663068. Antimicrob. Agents Chemother. 56, 3498-3507 (2012).

20. Herschhorn, A. et al. The beta20-beta21 of gp120 is a regulatory switch for HIV-1 Env conformational transitions. Nat. Commun. 8, 1049 (2017).

21. Sanders, R. W. et al. A next-generation cleaved, soluble HIV-1 Env trimer, BG505 SOSIP.664gp140, expresses multiple epitopes for broadly neutralizing but not non-neutralizing antibodies. PLoS Pathog. 9, e1003618 (2013).

22. Garces, F. et al. Affinity maturation of a potent family of HIV antibodies is primarily focused on accommodating or avoiding glycans. Immunity $\mathbf{4 3}$, 1053-1063 (2015)

23. Strong, M. et al. Toward the structural genomics of complexes: crystal structure of a PE/PPE protein complex from Mycobacterium tuberculosis. Proc. Natl Acad. Sci. USA 103, 8060-8065 (2006).

24. Pancera, M. et al. Structure and immune recognition of trimeric pre-fusion HIV-1 Env. Nature 514, 455-461 (2014).

25. Guenaga, J. et al. Glycine substitution at helix-to-coil transitions facilitates the structural determination of a stabilized subtype C HIV envelope glycoprotein. Immunity 46, 792-803 e793 (2017).

26. Mastalerz, M. Crystal engineering: covalent crystal growth. Nat. Chem. 5, 810-811 (2013).

27. Hupp, J. T. Crystal engineering: towards artificial enzymes. Nat. Chem. 2, 432-433 (2010).

28. Thomas, J. M. Diffusionless reactions and crystal engineering. Nature $\mathbf{2 8 9}$, 633-634 (1981).

29. Zaworotko, M. J. Crystal engineering comes of age. Nat. Chem. 3, 653 (2011).

30. Lee, S., Kapustin, E. A. \& Yaghi, O. M. Coordinative alignment of molecules in chiral metal-organic frameworks. Science 353, 808-811 (2016).

31. Inokuma, Y. et al. X-ray analysis on the nanogram to microgram scale using porous complexes. Nature 495, 461-466 (2013).

32. Zheng, J. et al. From molecular to macroscopic via the rational design of a selfassembled 3D DNA crystal. Nature 461, 74-77 (2009).

33. Lanci, C. J. et al. Computational design of a protein crystal. Proc. Natl Acad. Sci. USA 109, 7304-7309 (2012).

34. Lai, Y. T. et al. Structure of a designed protein cage that self-assembles into a highly porous cube. Nat. Chem. 6, 1065-1071 (2014).

35. Little, M. A. et al. Trapping virtual pores by crystal retro-engineering. Nat. Chem. 7, 153-159 (2014).

36. Ghosh, E., Kumari, P., Jaiman, D. \& Shukla, A. K. Methodological advances: the unsung heroes of the GPCR structural revolution. Nat. Rev. Mol. Cell Biol. 16, 69-81 (2015).

37. Lieberman, R. L., Culver, J. A., Entzminger, K. C., Pai, J. C. \& Maynard, J. A. Crystallization chaperone strategies for membrane proteins. Methods 55, 293-302 (2011).

38. Dunbar, J. et al. SAbDab: the structural antibody database. Nucleic Acids Res. 42, D1140-D1146 (2014) 
39. Pardon, E. et al. A general protocol for the generation of Nanobodies for structural biology. Nat. Protoc. 9, 674-693 (2014).

40. Misasi, J. et al. Structural and molecular basis for Ebola virus neutralization by protective human antibodies. Science 351, 1343-1346 (2016).

41. Lee, P. S. et al. Receptor mimicry by antibody F045-092 facilitates universal binding to the H3 subtype of influenza virus. Nat. Commun. 5, 3614 (2014).

42. Meanwell, N. A. et al. Inhibitors of HIV-1 attachment. Part 2: an initial survey of indole substitution patterns. Bioorg. Med. Chem. Lett. 19, 1977-1981 (2009).

43. Wang, T. et al. Discovery of 4-benzoyl-1-[(4-methoxy-1H- pyrrolo[2,3-b] pyridin-3-yl)oxoacetyl]-2-(R)-methylpiperazine (BMS-378806): a novel HIV-1 attachment inhibitor that interferes with CD4-gp120 interactions. J. Med. Chem. 46, 4236-4239 (2003).

44. Li, M. et al. Human immunodeficiency virus Type 1 env clones from acute and early subtype B infections for standardized assessments of vaccine-elicited neutralizing antibodies. J. Virol. 79, 10108-10125 (2005).

45. Seaman, M. S. et al. Tiered categorization of a diverse panel of HIV-1 Env pseudoviruses for assessment of neutralizing antibodies. J. Virol. 84, 1439-1452 (2010).

46. Otwinowski, Z. \& Minor, W. Processing of X-ray diffraction data collected in oscillation mode. Methods Enzymol. 276, 307-326 (1997).

47. Collaborative Computational Project, N.. The CCP4 suite: programs for protein crystallography. Acta Crystallogr. D Biol. Crystallogr. 50, 760-763 (1994).

48. Emsley, P. \& Cowtan, K. Coot: model-building tools for molecular graphics. Acta Crystallogr. D Biol. Crystallogr. 60, 2126-2132 (2004).

49. Adams, P. D. et al. PHENIX: a comprehensive Python-based system for macromolecular structure solution. Acta Crystallogr. D Biol. Crystallogr. 66 213-221 (2010).

50. Delano, W. L. The PyMOL Molecular Graphics System (DeLano Scientific, San Carlos, CA, 2002).

\section{Acknowledgements}

We thank D. Burton and M. Fineberg for the sequences of NAC-identified antibodies, including the 3H109L member of the PGT121 lineage used in crystallization, M. Connors for the sequence of antibody $35 \mathrm{O} 22$ used in crystallization, J. Stuckey for assistance with figures, and members of Structural Biology and Structural Bioinformatics Core Sections, Vaccine Research Center for discussions and comments on the manuscript and the WCMC/AMC/TSRI HIVRAD team for their contributions to the design and validation of near-native mimicry for soluble BG505 SOSIP.664 trimers. Funding was provided by the Intramural Research Program of the Vaccine Research Center, National Institute of Allergy and Infectious Diseases, the Intramural AIDS Targeted Antiretroviral Program, the National Institute of General Medical Sciences, National Institutes of Health. Use of sector 22 (Southeast Region Collaborative Access team) at the Advanced Photon Source was supported by the US Department of Energy, Basic Energy Sciences, Office of Science, under contract no. W-31-109-Eng-38.

\section{Author contributions}

Y.-T.L. determined the crystal structures with assistance from D.R.L. and P.D.K. S.O., M. K.L., B.L., K.M., R.T.B., N.A.D.-R. and J.R.M. performed virus neutralization assays. A.S. performed and analyzed ITC experiments. C.S.C., A.D., D.P., Y.Y. and B.Z. expressed and purified proteins. T.W., A.H. and J.S. synthesized small-molecule compounds. G.-Y. C. analyzed virus resistance data. Y.-T.L. and P.D.K. analyzed the data and wrote the paper.

\section{Additional information}

Supplementary Information accompanies this paper at https://doi.org/10.1038/s41467018-07851-1.

Competing interests: D.R.L. and T.W. own stock in Bristol-Myers Squibb. All other authors declare no competing interests.

Reprints and permission information is available online at http://npg.nature.com/ reprintsandpermissions/

Publisher's note: Springer Nature remains neutral with regard to jurisdictional claims in published maps and institutional affiliations.

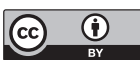

Open Access This article is licensed under a Creative Commons Attribution 4.0 International License, which permits use, sharing, adaptation, distribution and reproduction in any medium or format, as long as you give appropriate credit to the original author(s) and the source, provide a link to the Creative Commons license, and indicate if changes were made. The images or other third party material in this article are included in the article's Creative Commons license, unless indicated otherwise in a credit line to the material. If material is not included in the article's Creative Commons license and your intended use is not permitted by statutory regulation or exceeds the permitted use, you will need to obtain permission directly from the copyright holder. To view a copy of this license, visit http://creativecommons.org/ licenses/by/4.0/.

(c) The Author(s) 2019 